JOLRNAI. OF RESEARCH of the National Bureau of Standards-B. Mathematical Sciences

Vol. 79B, Nos. I and 2, January-June 1975

\title{
A Note on the Metrizability of Spaces With Countably Based Closed Sets
}

\author{
Ralph R. Sabella \\ California State University, Northridge, California 91324 \\ (January 14, 1975)
}

\begin{abstract}
The main result of this note is a generalization of an earlier theorem on the metrizability of spaces with countably based closed sets. Use is made of some results related to co-convergent spaces which are spaces having countably based compact sets.
\end{abstract}

Key words: Co-convergent; contra-convergent; Nagata spaces: open neighborhood assignments; stratifiable spaces; U-linked sequences.

C. E. Aull in $[1]^{1}$ defines a $D_{1}$-space as a topological space in which every closed set $F$ has a countable local base $\left\{B_{n}(F)\right\}$ : i.e., for each $n \in N$, the set of positive integers, $B_{n}(F)$ is an open set containing $F$; and if $F \subset R$, where $R$ is open, then $B_{k}(F) \subset R$ for some $k \in N$.

An open neighborhood assignment (ONA) is defined in [2] as a function

$$
U: X \times N \rightarrow U\{N(x): x \in X\}
$$

such that $x \in U(x, n) \equiv U_{n}(x)$ where $X$ is a topological space and $N(x)$ is the collection of open neighborhoods of $x$. If $U$ is an ONA then the sequence $\left\{y_{n}\right\}$ is $U$-linked to $\left\{x_{n}\right\}$ if $y_{n} \in U_{n}\left(x_{n}\right)$ for all $n$. Using the notation $C p\left\{x_{n}\right\}$ for the set of cluster points of $\left\{x_{n}\right\}$ we define a space to be $c o$. convergent (contra-convergent) if $C p\left\{x_{n}\right\} \subset C p\left\{y_{n}\right\} \quad\left(C p\left\{y_{n}\right\} \subset C p\left\{x_{n}\right\}\right)$ whenever $\left\{y_{n}\right\}$ is $U$-linked to $\left\{x_{n}\right\}$. If on $X$ there is an ONA $U$ having some property $P$ we shall say " $X$ is $P$ " or " $U$ is $P$." Finally for any $S \subset X$ and ONA $U$ we have $U_{n}(S) \equiv \cup\left\{U_{n}(x): x \in S\right\}$.

The following two theorems were proved in [2]:

THEоRем 1: $\mathrm{X}$ is metrizable iff it is a co-convergent, contra-convergent $T_{0}$-space.

THEOREM 2: The following are equivalent on a space X:

(a) $\mathrm{X}$ is co-convergent.

(b) There exists an ONA $\mathrm{U}$ on $\mathrm{X}$ such that for any countably compact $\mathrm{K}$ and open $\mathrm{R}$ containing $\mathrm{K}, \mathrm{U}_{\mathrm{n}}(\mathrm{K}) \subset \mathrm{R}$ for some $\mathrm{n} \epsilon \mathrm{N}$. 
(c) There exists an $O N A \mathrm{U}$ on $\mathrm{X}$ such that for any convergent sequence $\left\{\mathrm{x}_{\mathrm{n}}\right\}$ with limit $\mathrm{x}_{0}$ and open $\mathrm{R}$ containing $\left\{\mathrm{x}_{\mathbf{k}}: \mathrm{k}=0,1, \ldots\right\}, \mathrm{U}_{\mathrm{n}}\left(\left\{\mathrm{x}_{\mathbf{k}}: \mathrm{k}=0,1, \ldots\right\}\right) \subset \mathrm{R}$ for some $\mathrm{n} \epsilon \mathrm{N}$.

A characterization of a natural- $D_{1}$ space is as a space $X$ on which there is an ONA $U$ such that for every closed set $F,\left\{U_{n}(F)\right\}$ is a local base for $F$. We note that if $X$ is $T_{2}$ and $U$ is a natural- $D_{1}$ ONA then it is co-convergent.

We have an analogue of Theorem 2:

THEOREM 3: Let $\mathrm{U}$ be an ONA on $\mathrm{X}$. Then the following are equivalent:

(a) $\mathrm{U}$ is natural- $\mathrm{D}_{1}$. $1,2, \ldots\}$.

(b) For any sequence $\left\{\mathbf{x}_{\mathbf{k}}\right\},\left\{\mathrm{U}_{\mathbf{n}}\left(\Delta\left\{\mathbf{x}_{\mathbf{k}}\right\}\right)\right.$ is a local base for $\Delta\left\{\mathbf{x}_{\mathbf{k}}\right\}$ where $\Delta\left\{\mathbf{x}_{\mathbf{k}}\right\} \equiv \operatorname{Cl}\left\{\mathbf{x}_{\mathbf{n}}: \mathbf{n}=\right.$

Proof: Only (b) implies (a) requires any consideration. Suppose $F$ is closed and is contained in an open $R$. If for each $n$ there is a $z_{n} \epsilon U_{n}(F)-R$ then there is a sequence $\left\{x_{n}\right\}$ in $F$ with $\left\{z_{n}\right\} U$-linked to $\left\{x_{n}\right\}$. But then $\Delta\left\{x_{n}\right\} \subset F$ and for some $k, U_{k}\left(\Delta\left\{x_{n}\right\}\right) \subset R$ implying the contradiction $z_{k} \notin R$.

The following result was proved in [3]:

THEOREM 4: If $\mathrm{X}$ has at most a finite number of isolated points it is compact and metrizable iff it is natural $-\mathrm{D}_{1}$ and Hausdorff.

We shall generalize Theorem 4, by using Aull's result in [1] that every regular, $D_{1}$-space is the union of a countably compact set and a set of isolated points.

THEOREM 5: $\mathrm{X}$ is compact and metrizable iff it is natural- $\mathrm{D}_{1}$ and Hausdorff,

PROOF: We need only consider the sufficiency part of the proof, the necessary part being the same as for Theorem 4 .

Let $U$ be a natural- $D_{1}$ ONA on $X$. Without loss of generality we can assume $U$ is nested. Furthermore $\left\{U_{n}(x)\right\}$ is a local base for each $x \in X . X$ is regular, for if $F$ is closed and $x \in F$ and if for all $n$ there is a $z_{n} \in U_{n}(x)-U_{n}(F)$, then there is a sequence $\left\{y_{n}\right\}$ in $F$ such that $\left\{z_{n}\right\}$ is $U$-linked to $\left\{y_{n}\right\}$. It follows that $\left\{z_{n}\right\}$ converges to $x$. Hence there is an $M>0$ such that for all $k$ $>M, z_{\mathbf{k}} \in X-F$. Since $U$ is natural- $D_{1}$ there is an $n>M$ such that $U_{n}(F) \subset X-\Delta\left\{z_{\mathbf{k}}\right\}_{\mathbf{k}=M}^{\infty}$, which implies $U_{n}\left(y_{n}\right) \subset X-\Delta\left\{z_{\boldsymbol{k}}\right\}_{\boldsymbol{k}=M}^{\infty}$, contradicting $z_{n} \in U_{n}\left(y_{n}\right)$.

Let $X=C \cup I$ where $C$ is countably compact and $I$ is a set of isolated points of $X$. We can assume $C \cap I=0$. Again without loss of generality we can let $U_{n}(x)=\{x\}$ for all $x \in I$ and all $n$. Let $\left\{y_{n}\right\}$ be $U$-linked to $\left\{x_{n}\right\}$ and $y \in C p\left\{y_{n}\right\}$. Then there is a subsequence $\left\{y_{n_{k}}\right\}$ of $\left\{y_{n}\right\}$ converging to $y$. If $x_{n_{k}} \in I$ for infinitely many $k$, then $\left\{x_{n_{k}}\right\}$ clusters at $y$. If $\left\{x_{n_{k}}\right\} \epsilon C$ for infinitely many $k,\left\{x_{n_{k}}\right\}$ clusters at some $x \in C$, implying by the co-convergence of $U$ that $x=y$. Hence $U$ is contra-convergent and by Theorem $1, X$ is metrizable.

\section{References}

[1] Aull, C. E., Closed Set Countability Axioms, Indag. Math. 28, 311-316 (1966).

[2] Sabella, R. R., Convergence Properties of Neighboring Sequences, Proc. Amer. Math. Soc. 38, 405-409 (1973).

[3] Sabella, R. R., Metrizability of Spaces with Countably Based Closed Sets, Port. Math. 29, 1-3 (1970).

[4] Sabella, R. R., Spaces in Which Compact Sets have Countable Local Bases, Proc. Amer. Math. Soc. (to appear).

(Paper 79B1\&2-422) 